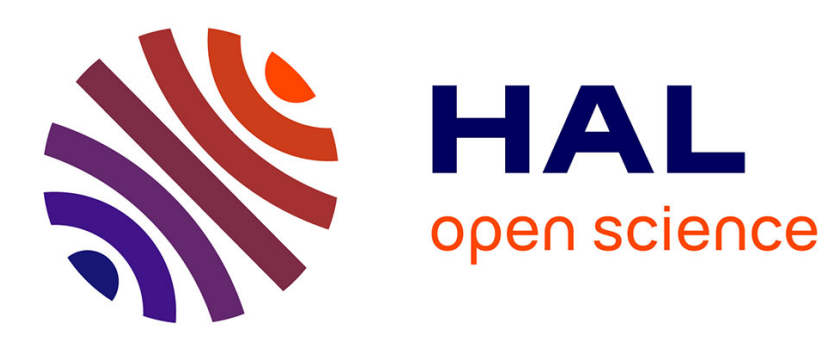

\title{
Acoustic modulators for optical fibres
}

G.S. Kino, W.P. Risk

\section{- To cite this version:}

G.S. Kino, W.P. Risk. Acoustic modulators for optical fibres. Revue de Physique Appliquée, 1985, 20

(6), pp.333-340. 10.1051/rphysap:01985002006033300 . jpa-00245341

\section{HAL Id: jpa-00245341 \\ https://hal.science/jpa-00245341}

Submitted on 1 Jan 1985

HAL is a multi-disciplinary open access archive for the deposit and dissemination of scientific research documents, whether they are published or not. The documents may come from teaching and research institutions in France or abroad, or from public or private research centers.
L'archive ouverte pluridisciplinaire HAL, est destinée au dépôt et à la diffusion de documents scientifiques de niveau recherche, publiés ou non, émanant des établissements d'enseignement et de recherche français ou étrangers, des laboratoires publics ou privés. 
Classification

Physics Abstracts

$78.20 \mathrm{H}-42.30 \mathrm{~L}$

\title{
Acoustic modulators for optical fibres
}

\author{
G. S. Kino and W. P. Risk \\ Edward L. Ginzton Laboratory, Stanford University, Stanford, Ca 94305, U.S.A.
}

(Reçu le 7 septembre 1984, accepté le 16 novembre 1984)

\begin{abstract}
Résumé. - Nous avons examiné des techniques destinées à la modulation acoustique dans une fibre optique. Une théorie simple de modes couplés a été développée; elle montre qu'en utilisant une onde acoustique se propageant dans la direction de la fibre, il est possible de réaliser un modulateur à bande latérale unique. Des résultats expérimentaux confirmant ces idées sont présentés ainsi que quelques idées sur les directions futures de ce travail.
\end{abstract}

\begin{abstract}
We have examined techniques for acoustic modulation of an optical fibre. A simple coupled mode theory has been developed which shows that by use of an acoustic wave propagating in the direction of the fibre, it is possible to make a single-sideband modulator. Experimental results confirming these ideas are given, along with some ideas for future direction of this work.
\end{abstract}

\section{Introduction.}

Interest in the use of optical fibres for sensing and communications systems has spurred the development of a wide variety of single-mode fibre optic components [1, 2], which perform the same functions as their more familiar counterparts in bulk optics. Among the optical components produced in fibre form are polarizers, beamsplitters, polarization controllers, filters, and phase-modulators. One important component which has proved difficult to produce in fibre form is the single-sideband frequency-shifter, which counterpart in bulk optics is the Bragg cell. The only successful attempts to date to create such a device have relied on polarization coupling in birefringent optical fibre. Nosu et al. [3] used two small PZT cylinders to create an asymmetric stress in a relatively low birefringence fibre (beat length $=3.4 \mathrm{~cm}$ ) to cause coupling between its two polarizations. By spacing the cylinders three-quarters of a beat length apart and driving them $90^{\circ}$ out-of-phase, they were able to cancel one sideband and reinforce the other.

While this technique is interesting, it is not suitable for fibres with very short beat lengths and may be limited in modulation frequency and in suppression of undesired sidebands. We report here on what we believe to be a more attractive method, namely the use of a travelling acoustic wave to produce coupling between the two polarizations of birefringent fibre. The coupling takes place continuously over a certain length; rather than at discrete points (as in the Nosu device).
Also, the phase relationship between each infinitesimal section of the coupling region required to reinforce one sideband and cancel the other is guaranteed by the propagating nature of the acoustic wave. More specifically, an optical wave with frequency $\omega_{1}$ and propagation constant $k_{1}$ polarized along one principle axis of birefringent fibre interacts with an acoustic wave having frequency $\omega_{\mathrm{a}}$ and propagation constant $k_{\mathrm{a}}$ to produce a second optical wave polarized along the orthogonal principle axis with frequency $\omega_{2}=$ $\omega_{1} \pm \omega_{\mathrm{a}}$ and propagation constant given by $k_{2}=$ $k_{1}+k_{\mathrm{a}}$, as shown in figure 1. This latter condition expresses a phase-matching requirement between the

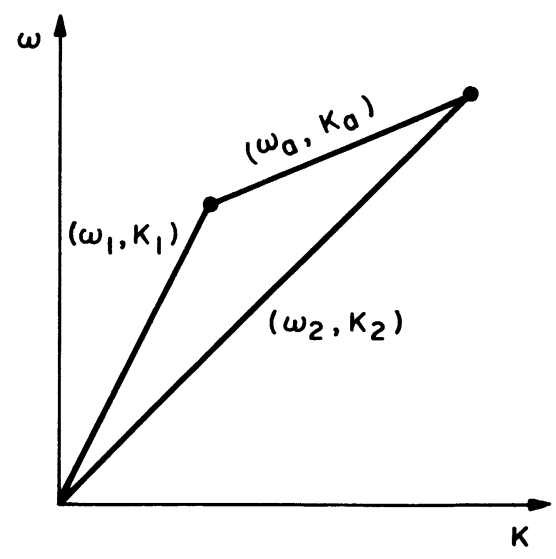

Fig. 1. - Phase-matching between the two optical waves and the acoustic wave. 
acoustic waves and the two optical waves, while the former condition expresses the fact that a frequency shift will be obtained in the generated optical wave.

It will be observed that if $k_{2}>k_{1}$ and the acoustic wave is travelling in the forward direction $\omega_{\mathrm{a}} / k_{\mathrm{a}}$ is positive and the optical wave is upshifted in frequency. On the other hand, if the acoustic wave is travelling in the backward direction $\omega_{\mathrm{a}} / k_{\mathrm{a}}$ is negative and the optical wave is downshifted in frequency. Finally, if the fibre is vibrated at frequency $\omega_{0}$ by a spatially periodic stress field which can be regarded as a standing wave, only the space harmonic components with a wave number $k_{0}$ such that $k_{2}=k_{1}+k_{0}$ will give strong interactions. As there will be both forward and backward wave space harmonics with $\omega_{0} / k_{0}$ positive and negative respectively, two optical frequencies $\omega_{1}+\omega_{0}$ and $\omega_{1}-\omega_{0}$ respectively and satisfying $k_{2}=k_{1}+k_{0}$ will be generated. For example, both sidebands will be generated by the periodically ridged block illustrated in figure 2 , which is placed in contact with the fibre and vibrated at a frequency $\omega_{0}$ [4].

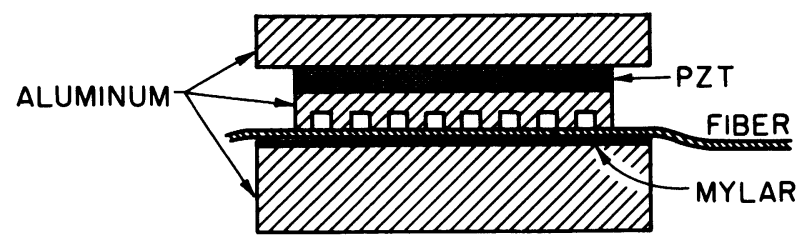

Fig. 2. - Modulator of reference [4], consisting of a periodically ridged block vibrated on top of a single-mode birefringent fibre.

We present a simple coupled-mode analysis describing the important characteristics of these frequency shifters, determine the conditions for optimum coupling, and derive an expression for the modulation bandwidth. We show that higher modulation frequencies can be obtained by allowing the acoustic wave to interact with the fibre at an angle and consider the extension of our coupled-mode model to this case, showing that the simple model is adequate for all frequencies of present interest. Also, we briefly consider coupling due to space harmonics of the perturbing stress field to illustrate that devices of the kind depicted in figure 2 can be treated by the same type of coupledmode theory. Finally, we present the results of two preliminary experiments which illustrate these ideas, and discuss the design of future devices suggested by the outcome of these experiments.

\section{Theory.}

A « single-mode» optical fibre will support two propagating modes which have orthogonal linear polarizations in the weakly-guiding approximation [5]. We can take these linear polarizations as being along the $x$ - and $y$-axes in figure 3 . In ideal, perfectly symmetric fibre, these modes are degenerate, that is, their propagation constants $k_{x}$ and $k_{y}$ are equal. Any polarization state launched into the fibre will propagate unaltered. In real fibre, however, geometrical asymmetries and residual stresses can break the degeneracy of the $x$-and $y$-polarizations so that $k_{x}$ and $k_{y}$ are slightly different. Random perturbations along the fibre can then cause coupling between these two modes and a polarization state launched into the fibre will generally be maintained. Hence, ordinary single-mode fibre will not usually preserve the input polarization. To circumvent this problem, the fibre can be made birefringent intentionally (e.g., by making the core elliptical [6] or by incorporating asymmetric stress into the fibre during fabrication [7]) so that the difference between $k_{x}$ and $k_{y}$ is relatively large. This will largely eliminate the coupling between the two polarizations and introduce two principle axes which we can take as being along $x$ and $y$. The two waves which are linearly polarized along the principle axes will be unaltered in polarization state as they propagate along the fibre. If any other polarization is launched in the fibre, it will decompose into a superposition of these

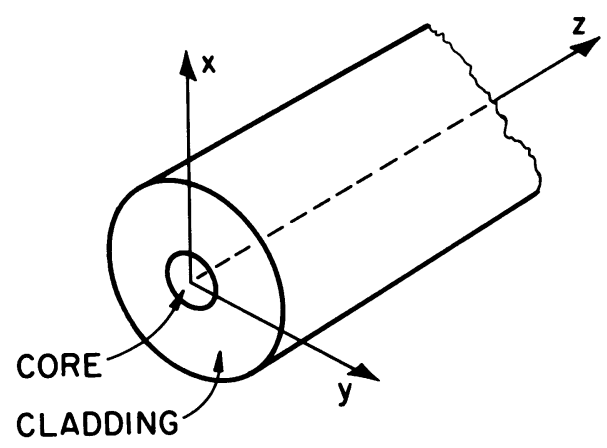

Fig. 3. - Single mode fibre and the $x-y-z$ coordinate system.

two linear polarizations. Because of the difference between $k_{x}$ and $k_{y}$, these two components will develop a phase difference as they propagate down the fibre, and the state of polarization will change. When the phase difference between the two polarizations is an integer multiple of $2 \pi$, the original polarization state will be reproduced. The distance required for this phase difference of $2 \pi$ is called the beat length $L_{B}$, where $L_{\mathrm{B}}=\lambda_{0} / \Delta n$ and $\Delta n=\left|n_{x}-n_{y}\right|$.

Although the two polarizations in birefringent fibre are normally uncoupled because of the relatively large difference in propagation constant, coupling can be produced between them by perturbing the birefringence of the fibre. For example, stressing the fibre by twisting [8] or squeezing [9] it can produce such coupling. When a perturbation which causes coupling is applied to the fibre, the effect of that coupling on the two polarizations can be described by the following 
set of coupled equations for the complex mode amplitudes $A_{1}$ and $A_{2}$ :

$$
\begin{aligned}
& \frac{\mathrm{d} A_{1}}{\mathrm{~d} z}=-j\left(k_{1}+\kappa_{11}\right) A_{1}+\kappa_{21} A_{2} \\
& \frac{\mathrm{d} A_{2}}{\mathrm{~d} z}=\kappa_{12} A_{1}-j\left(k_{2}+\kappa_{22}\right) A_{2}
\end{aligned}
$$

where mode 1 is represented as $A_{1} \mathrm{e}^{j \omega_{1 t} t}=a_{1} \mathrm{e}^{j\left(\omega_{11}-k_{12}\right)}$ and mode 2 is represented as $A_{2} \mathrm{e}^{j \omega_{2 t} t}=a_{2} \mathrm{e}^{j\left(\omega_{2} t-k_{2} z\right)}$. The propagation constants of the uncoupled modes are $k_{1}=2 \pi n_{1} / \lambda_{0}$ and $k_{2}=2 \pi n_{2} / \lambda_{0} \cdot \kappa_{11}, \kappa_{22}$ represent the reaction of the coupling process on the 1 , 2 modes respectively; and $\kappa_{12}, \kappa_{21}$ represent the crosscoupling from mode 1 to 2 and from mode 2 to 1 respectively. For most cases of interest, $k_{1} \gg \kappa_{11}$ and $k_{2} \gg \kappa_{22}$. Furthermore, the mode amplitudes $A_{1}$ and $A_{2}$ must satisfy conservation of power as expressed by the Manley-Rowe relation :

$$
\frac{1}{\omega_{1}} \frac{\mathrm{d}\left(A_{1} A_{1}^{*}\right)}{\mathrm{d} z}=-\frac{1}{\omega_{2}} \frac{\mathrm{d}\left(A_{2} A_{2}^{*}\right)}{\mathrm{d} z} .
$$

This requirement gives the condition that

$$
\kappa_{21}=-\frac{\omega_{1}}{\omega_{2}} \kappa_{12}^{*} .
$$

Hence, equation (2) can be written as

$$
\begin{aligned}
& \frac{\mathrm{d} A_{1}}{\mathrm{~d} z}=-j k_{1} A_{1}-\frac{\omega_{1}}{\omega_{2}} \kappa_{12}^{*} A_{2} \\
& \frac{\mathrm{d} A_{2}}{\mathrm{~d} z}=-j k_{2} A_{2}+\kappa_{12} A_{1} .
\end{aligned}
$$

Since for all cases of interest here, $\omega_{\mathrm{a}} \ll \omega_{1}, \omega_{2}$, we can take $\omega_{2} \approx \omega_{1}$ and replace $\omega_{1} / \omega_{2}$ by 1 in equation (3a).

The coupling coefficient $\kappa$ is related to the perturbing birefringence and can be found explicitly for a variety of simple cases [10]. For example, the coupling coefficient for squeezing a fibre between two flat plates can be found in terms of the force per unit length applied and is uniform with distance. We are presently deriving a similar relationship for the acoustic case, giving the coupling coefficient in terms of the applied acoustic power and material parameters, but for the purposes of this discussion, it suffices to use $\kappa$ directly.

We now assume a travelling wave form for the coupling coefficient, i.e., $\kappa_{12}(z)=\kappa_{0} \mathrm{e}^{-j \boldsymbol{k}_{\mathrm{a}} z}$. This form is then substituted into equation (3) and using the definitions of $A_{1}, A_{2}$, we obtain :

$$
\begin{aligned}
& \frac{\mathrm{d} a_{1}}{\mathrm{~d} z}=-\kappa_{0}^{*} \mathrm{e}^{j \Delta k z} a_{2} \\
& \frac{\mathrm{d} a_{2}}{\mathrm{~d} z}=\kappa_{0} \mathrm{e}^{-j \Delta k z} a_{1}
\end{aligned}
$$

where $\Delta k=k_{\mathrm{a}}+k_{1}-k_{2}, k_{\mathrm{a}}$ is the propagation cons- tant of the acoustic wave given by $k_{\mathrm{a}}=2 \pi / \Lambda$, and $\Lambda$ is the acoustic wavelength. If we assume boundary conditions at $z=0$ of $a_{2}=0$ and $a_{1}=1$, then the solution is [11] :

$$
\begin{aligned}
a_{1}(z) & =\exp \left(\frac{j \Delta k z}{2}\right)\left(\cos \left(\frac{1}{2} \sqrt{4\left|\kappa_{0}\right|^{2}+(\Delta k)^{2}} z\right)-\right. \\
& \left.-j \frac{\Delta k}{\sqrt{4\left|\kappa_{0}\right|^{2}+(\Delta k)^{2}}} \sin \left(\frac{1}{2} \sqrt{4\left|\kappa_{0}\right|^{2}+(\Delta k)^{2}} z\right)\right)
\end{aligned}
$$

$$
\begin{array}{r}
a_{2}(z)=\exp \left(\frac{-j \Delta k z}{2}\right) \frac{2 \kappa_{0}}{\sqrt{4\left|\kappa_{0}\right|^{2}+(\Delta k)^{2}}} \times \\
\times \sin \left(\frac{1}{2} \sqrt{4\left|\kappa_{0}\right|^{2}+(\Delta k)^{2}} z\right) .
\end{array}
$$

Defining the power in mode 1 as $P_{1}=a_{1} a_{1}^{*}$ and the power in mode 2 as $P_{2}=a_{2} a_{2}^{*}$, the cross-coupled power can be expressed as :

$\frac{P_{2}(z)}{P_{1}(0)}=\frac{4\left|\kappa_{0}\right|^{2}}{4\left|\kappa_{0}\right|^{2}+(\Delta k)^{2}} \sin ^{2}\left(\frac{1}{2} \sqrt{4\left|\kappa_{0}\right|^{2}+(\Delta k)^{2}} z\right)$.

For example, suppose the coupling coefficient is constant along the fibre (i.e., $k_{\mathrm{a}}=0$ ) the power transfer is maximum at :

$$
L=\frac{\pi}{\sqrt{4\left|\kappa_{0}\right|^{2}+\left(k_{2}-k_{1}\right)^{2}}}
$$

and

$$
\frac{P_{2}(L)}{P_{1}(0)}=\frac{4\left|\kappa_{0}\right|^{2}}{4\left|\kappa_{0}\right|^{2}+\left(k_{2}-k_{1}\right)^{2}} .
$$

This amount of coupling is usually small because $\kappa_{0} \ll \frac{\left|\left(k_{2}-k_{1}\right)\right|}{2}$. Greater power transfer between the two polarisations can be obtained if the perturbation is applied periodically along the length of the fibre. If the spatial period of the perturbation is equal to the beat length of the fibre, the contributions to the coupled power from each infinitesimal segment along the fibre will add coherently and a large fraction of the power in one polarization can be transferred to the other. For example, squeezing the fibre by statically pressing on it with a ridged plate [9] or introducing a periodic rotation of the principle axes by rocking the fibre as it is pulled [12] can produce this cumulative coupling.

Hence, if the wavelength $\Lambda$ of the travelling acoustic wave coupling the two optical modes equals the beat length of the fibre $L_{\mathrm{B}}$, then $\Delta k=0$ and equation (6) becomes :

$$
\frac{P_{2}(z)}{P_{1}(0)}=\sin ^{2}\left(\left|\kappa_{0}\right| z\right) .
$$


If the acoustic wave interacts with the fibre over a length $L$, and if the acoustic power is adjusted so that $\left|\kappa_{0}\right| L=\pi / 2$, then all of the power in mode 1 will be transferred to mode 2 . As mentioned above, the "conservation of momentum " or phase-matching condition $k_{2}=k_{1}+k_{\mathrm{a}}$ must be satisfied for optimum power transfer. In addition, the «conservation of energy " condition $\omega_{2}=\omega_{1}+\omega_{\mathrm{a}}$ must also be satisfied.

We can now apply the preceding analysis to a particular case. Suppose we have a birefringent fibre as in figure 3 and that the $x$-and $y$-axes are chosen so that the $x$-axis coincides with the slow polarization and the $y$-axis coincides with the fast polarization, i.e. $k_{x}>k_{y}$. All the light is initially in the fast polarization, which we identify with mode 1 in the foregoing analysis. If the phase-matching requirement $k_{x}=k_{y}+k_{\mathrm{a}}$ is satisfied, the light coupled from the fast polarization (mode 1) to the slow polarization (mode 2) will be upshifted in frequency according to the relationship $\omega_{x}=\omega_{y}+\omega_{\mathrm{a}}$. Conversely, if light is initially only in the slow polarization (mode 2 ) it will be coupled to the fast polarization (mode 1) with a downshift.

In the preceding analysis, the acoustic wave propagated in the same direction as the two optical beams in the fibre. Coupling can also occur if the acoustic wave propagates in the direction opposite to the two optical beams. Then light launched in the fast polarization will be coupled to the slow polarization with a downshift and light launched in the fast polarization will be coupled to the slow mode with an upshift. (These relationships are also evident from an $\omega-k$ diagram [13].)

An alternative way of stating this is that if light is launched only in the fast polarization, forward and backward travelling acoustic waves will produce upper and lower sidebands, respectively, in the slow polarization. If light is launched solely in the slow polarization, forward and backward travelling acoustic waves produce lower and upper sidebands, respectively, in the fast polarization. A single-sideband frequency shifter could therefore work as follows : a polarizer at the input ensures that only one of the two polarizations is initially launched; then an acoustic wave travelling in only one direction couples some or all of the light into the other polarization; finally, another polarizer ensures that only the frequency-shifted light is passed.

In a practical device, it will be desirable to vary the input frequency over some range and frequency modulate the shifted wave. As the frequency is detuned from the value which satisfies the phase-matching condition, the coupling efficiency will decrease. Using equation (6) with $\left|\kappa_{0}\right| L=\pi / 2$, we can solve for the value of $\Delta k$ for which $P_{2}(L) / P_{1}(0)$ drops to one-half. The full-width at half-maximum (FWHM) of the detuning curve is defined as twice this value of $\Delta k$. We obtain :

$$
\Delta k_{\mathrm{FWHM}} \approx \frac{2}{L} \sqrt{16-\pi^{2}}
$$

It is of greater practical interest to express this is terms of frequency, i.e., how far from phase-matching can the frequency be detuned before the coupling efficiency drops to one-half. The result is :

$$
\left(\frac{\Delta f}{f_{0}}\right)_{\mathrm{FWHM}} \approx 0.8 \frac{L_{\mathrm{B}}}{L}
$$

where $f_{0}$ is the phase-matched frequency. A good rule of thumb is that the fractional FWHM bandwidth is approximately the reciprocal of the number of beat lengths in the interaction region.

For an acoustic wave propagating along the fibre, having wavelength $\Lambda$ and speed of propagation $V_{\mathrm{a}}$, the phase-matching requirement dictates that $\Lambda=L_{\mathrm{B}}$ or that $f_{0}=V_{\mathrm{a}} / L_{\mathrm{B}}$. For commonly available fibre (beat lengths on the order of $1 \mathrm{~mm}$ ), and typical surface wave substrates (velocities of $3000-5000 \mathrm{~m} / \mathrm{s}$ ), the frequency shifts are limited to a few $\mathrm{MHz}$. Phasematching can be achieved at higher frequencies by allowing the acoustic wave to interact with the fibre at an angle, as shown in figure 4. Phase-matching

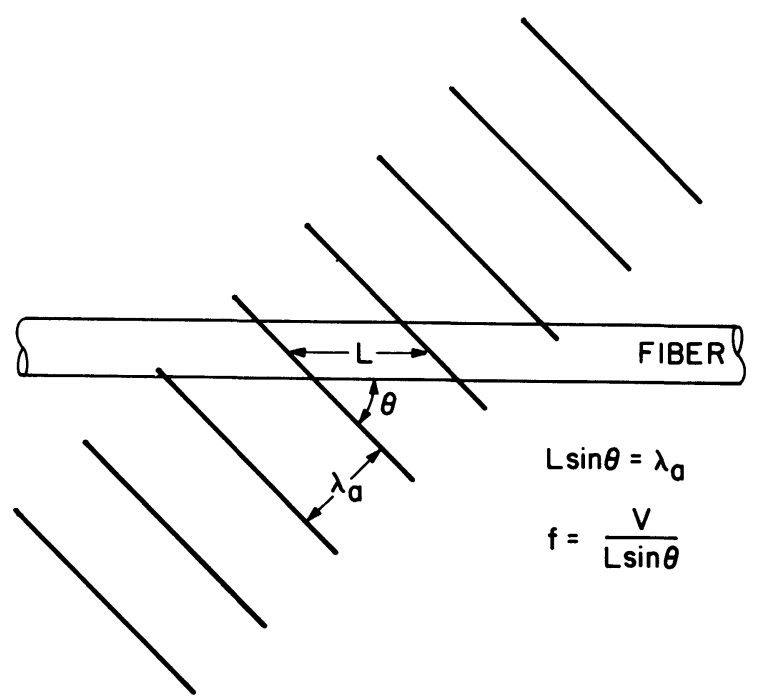

Fig. 4. - Phase-matching using an acoustic beam at an angle to the fibre.

between mode 1 having propagation constant $k_{1}$ and mode 2 have propagation constant $k_{2}>k_{1}$ will be satisfied when

$$
k_{1}+k_{\mathrm{a}} \sin \theta=k_{2} .
$$

The phase-matching angle is then given by

$$
\sin \theta=\frac{\Lambda}{L_{\mathbf{B}}} \text {. }
$$

Alternatively stated, the frequency at which phasematching occurs is given by

$$
f=\frac{V_{\mathbf{a}}}{L_{\mathrm{B}} \sin \theta} .
$$

Hence, phase-matching can be obtained at higher fre- 
quencies by decreasing the angle $\theta$. The preceding coupled mode theory can still be applied using an effective propagation constant $k_{\mathrm{a}, \text { eff }}=k_{\mathrm{a}} \sin \theta$.

Before discussing the experimental results, it is worth considering the effect on coupling produced by a more general stress field which is not necessarily a pure travelling wave. Two examples where such an approach is needed are that of a vibrating periodically ridged block in contact with the fibre and that of a series of periodically spaced elements in contact with the fibre which are excited by signals with a progressive phase delay between elements. The coupling coefficient arising from the latter type of stress field can be represented in the form :

$$
\kappa(z, t)=\mathrm{e}^{j\left(\omega_{\mathrm{a}} t-k_{\mathrm{a}} z\right)} \sum_{n=-\infty}^{\infty} \kappa_{n} \exp \left(-j \frac{2 n \pi z}{L}\right)+\text { c.c. } .
$$

Each space harmonic of the coupling coefficient couples the two optical waves with $\Delta k=k_{\mathrm{a}}+k_{1}-$ $k_{2}+2 n \pi / L$. The effectiveness of coupling due to each space harmonic can then be determined by this $\Delta k$ and equation (6).

For example, the case of figure 2 , where a ridged plate is statically pushed against the fibre and then vibrated about that point, can be analysed by taking account of all the space harmonic terms which excite the fibre at frequencies $\omega_{\mathrm{a}}$ and $-\omega_{\mathrm{a}}$. Putting $k_{\mathrm{a}}=0$ in equation (15) we obtain :

$\kappa(z, t)=\mathrm{e}^{j \omega_{\mathrm{a}} t} \sum_{n=-\infty}^{\infty} \kappa_{n} \exp \left(-j \frac{2 n \pi z}{L}\right)+$ c.c. .

If we choose the period $L$ equal to a beat length $L_{\mathrm{B}}$, the $n=0$ term does not have much effect, but the $n=1$ term of the first sum in equation (16) and the $n=-1$ term of the complex conjugate couple the two optical waves strongly. The $n=1$ term produces an upper sideband at $\omega+\omega_{\mathrm{a}}$ and the $n=-1$ term produces a lower sideband at $\omega-\omega_{\mathrm{a}}$. The higher order space harmonics will also produce coupling, but as noted earlier, their effect will be minor because they do not satisfy phase-matching.

\section{Experiment.}

We have conducted preliminary experiments to test the principles just discussed in section 2. The first experiment used a co-propagating surface acoustic wave to provide a $1.5 \mathrm{MHz}$ frequency shift, while the second experiment used a bulk longitudinal wave impinging on the fibre at an angle to provide a frequency shift of $15 \mathrm{MHz}$. In both cases, the sideband spectrum was observed by optically heterodyning the output of the frequency shifter down to a centre frequency of $40 \mathrm{MHz}$, using the system shown in figure 5 , so that the spectrum could be examined using a rf spectrum analyser. Although in principle a pure

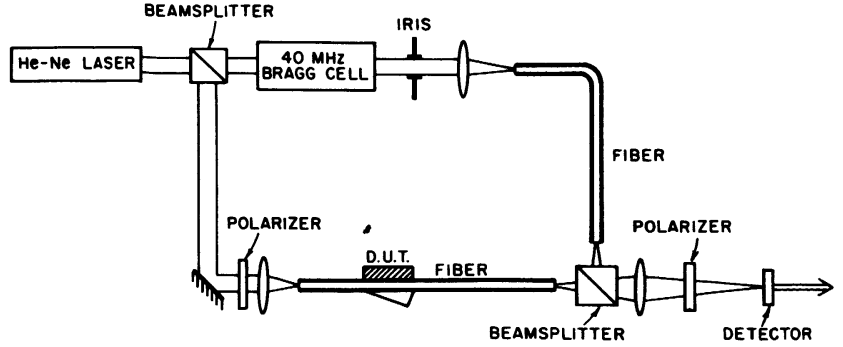

Fig. 5. - Optical heterodyning system used to evaluate the spectra of frequency shifting devices.

single-sideband frequency-shifted output is possible, in practice the selected polarization contains residual spectral components at the carrier frequency and at the frequency of the image sideband. These spurious signals can be caused by the finite extinction ratio of the polarizers, the limited polarization holding ability of the fibre, static coupling induced by the force used to press the fibre against the acoustic transducer, and by the fact that the true modes of the fibre are only approximately linearly polarized [14]. One goal of device design is the suppression of these undesired spectral components.

The first device to be described uses a surface acoustic wave launched by an edge-bonded transducer [15] onto an aluminum block to provide the required periodic perturbation of the fibre birefringence, as shown in figure 6 . The beam is $13 \mathrm{~mm}$ wide measured perpendicular to the fibre, so that much of the acoustic power is not effective in perturbing the fibre. This results in low efficiency, but can be corrected by using a more appropriate transducer design, as we will discuss later. Damping putty is applied to other surfaces of the block to extinguish reflections and waves launched in the wrong direction. The fibre is pressed against this surface by squeezing it between the block and a flat aluminum base. Although mass loading might be expected to damp the surface wave in the contact region, this has not proved to be the case. We believe this is because the actual region over which a Hertzian contact [16] is formed is very much smaller than an acoustic wavelength.

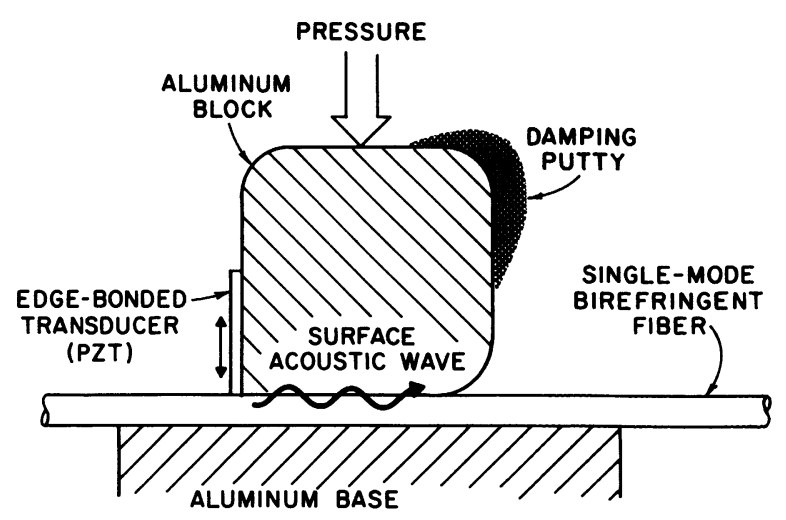

Fig. 6. - Frequency shifter using a surface wave launched by an edge-bonded transducer. 
The fiber was provided by Andrew Corp. and comes sheathed in a metallic coating and a protective plastic jacket, both of which are removed prior to using the fibre. The outer diameter of the stripped fibre is about $50 \mu \mathrm{m}$ and the birefringence results from the fibre's elliptical core (which measures roughly $1 \mu \mathrm{m}$ by $2 \mu \mathrm{m}$ ). The beat length of the fibre is $1.7 \mathrm{~mm}$ at an optical wavelength of $6328 \AA$.

When the transducer is driven at about $1.5 \mathrm{MHz}$, the acoustic wavelength is equal to the beat length of the fibre and the optimum coupling occurs. When driven with 5 watts of $\mathrm{CW}$ rf power, the output spectrum of figure 7 is obtained. Either an upper or lower sideband can be produced, depending on which polarization the input polarizer is aligned to excite. In both cases, the undesired sideband is suppressed by about $30 \mathrm{~dB}$. Suppression of the carrier requires more precise alignment of the polarizers than suppression of the unwanted sideband [17], and was not feasible in this first experiment where relatively coarse rotation stages were used. The maximum efficiency obtained with this device was around $10 \%$.

The second device to be discussed uses a bulk longitudinal wave impinging on the fibre at an angle to
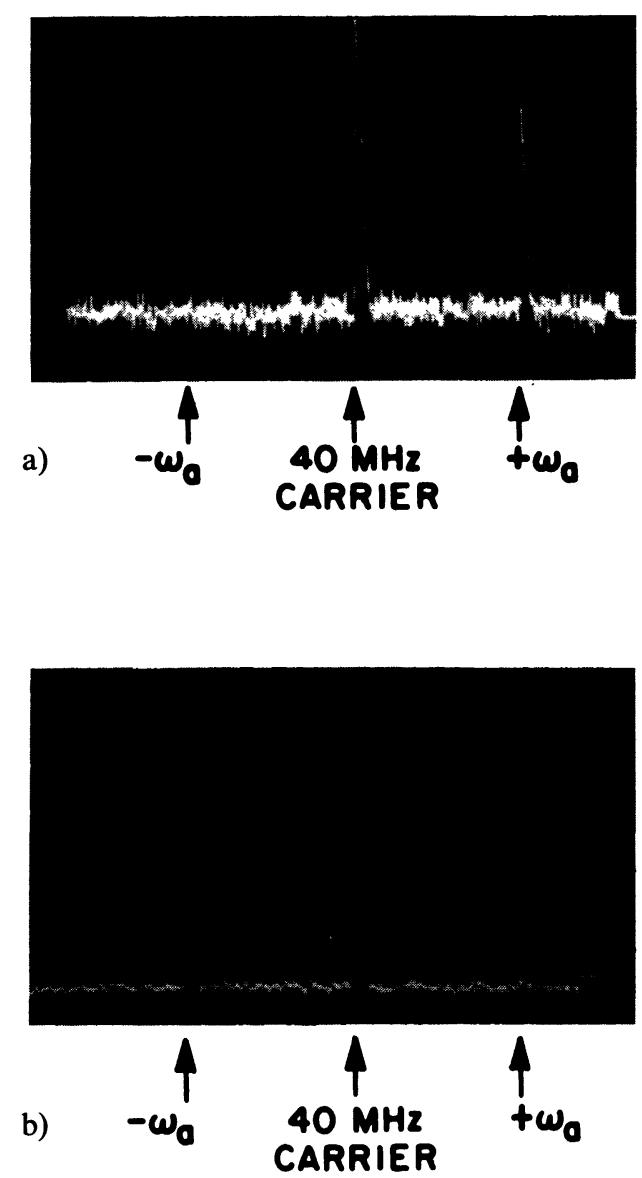

Fig. 7. - Output spectra of the device of figure 6. (a) Production of the upper sideband. Horiz. : $500 \mathrm{kHz} / \mathrm{div}$, Vert. : $10 \mathrm{~dB} / \mathrm{div}, 10$ averages. (b) Production of the lower sideband. Horiz. : $500 \mathrm{kHz} /$ div, Vert. : $10 \mathrm{~dB} / \mathrm{div}, 100$ averages. achieve a higher modulation frequency. As shown in figure 8, a PZT transducer is used to launch a bulk acoustic wave into a quartz wedge, angled so that the waves impinge on the fibre at $13.5^{\circ}$. This produces phase-matching at about $15 \mathrm{MHz}$. The beam size at the transducer is $1 \mathrm{~mm}$ by $10 \mathrm{~mm}$. The distance from the transducer to the fibre was chosen as a compromise between the phase variations of the near field and the beam divergence of the far field. Again, the fibre is pressed into contact with the quartz wedge by squeezing it between the wedge and a flat aluminum plate.

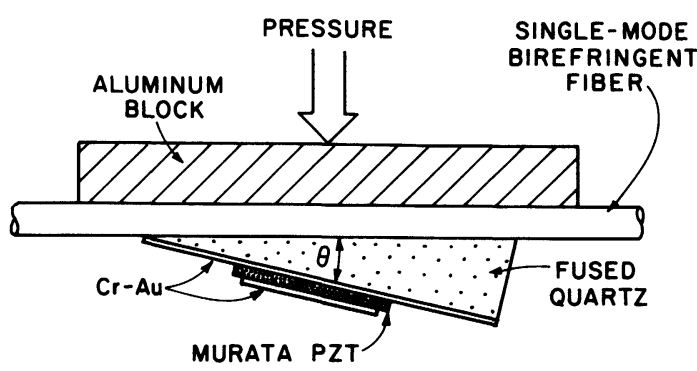

Fig. 8. - Frequency shifter using a bulk longitudinal wave impinging on the fibre at an angle.

When the transducer was driven with 2.25 watts of CW rf power, we obtained the sideband spectra shown in figure 9. In this experiment, care was taken to eliminate both the carrier and the unwanted sideband. In figure 9a, the output spectrum obtained with no output polarizer is shown, merely to mark the locations of the carrier and sidebands. In figures $9 b$ and $9 c$, the output polarizer has been replaced, and the two polarizers aligned to produce the lower sideband and upper sideband, respectively. The unwanted spectral components in both cases are suppressed by at least $20 \mathrm{~dB}$. The suppression may actually be greater since the suppressed components have disappeared into the noise floor of the system. The fractional FWHM bandwidth of this device is between $15 \%$ and $20 \%$, consistent with the prediction of coupled mode theory. The coupling efficiency is on the order of a few percent.

These devices have demonstrated that the basic principle of using an acoustic wave to dynamically couple the two polarizations of birefringent fibre and provide a frequency shift in the coupled wave is a valid one and that the behaviour of such a device is substantially as expected from the theory of section 2 . These present devices, while not practically useful for fibreoptic systems, do point the way toward more efficient and useful versions. We are presently constructing devices which use surface acoustic waves launched by interdigital transducers on $\mathrm{LiNbO}_{3}$ which will impinge on a fibre at an angle as shown in figure 10. Surface waves are desirable because the energy is confined to within roughly one wavelength of the surface. Also, since the acoustic beam crosses the fibre at an angle, all the energy in the beam should be effective in perturbing the fibre (unlike the surface wave device des- 
a)

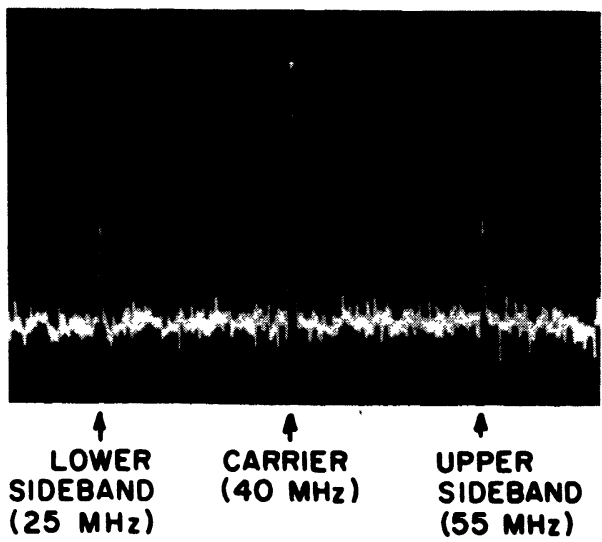

b)

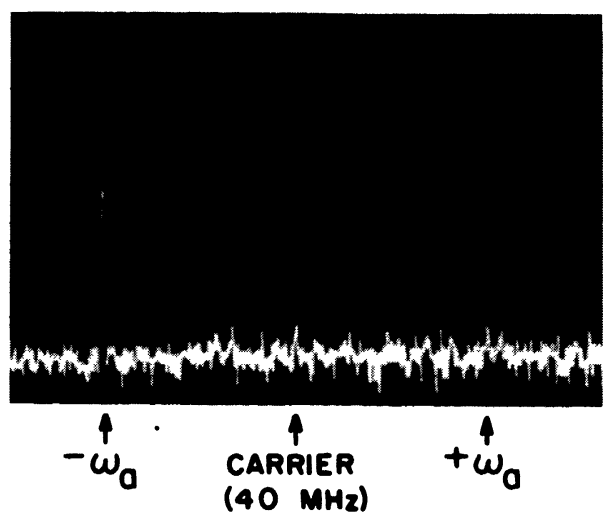

c)

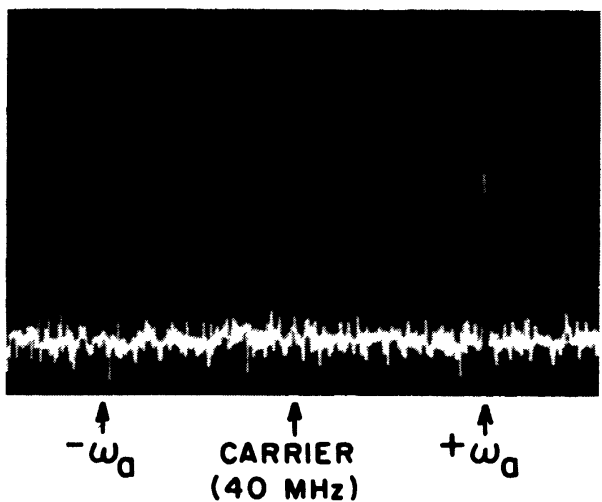

Fig. 9. - Output spectra of the device of figure 8. (a) Reference photo taking with no output polarizer to mark the location of the carrier and sidebands. (b) Production of the lower sideband. (c) Production of the upper sideband. (All photos : Horiz. : $5 \mathrm{MHz} /$ div, Vert. : $10 \mathrm{~dB} / \mathrm{div}, 10$ averages.)

cribed previously). Hence, these devices should produce greater coupling for the same amount of input power. Also, at a given fractional bandwidth, a larger absolute tuning range is obtained at a higher centre frequency, which can be obtained by increasing (i.e., moving toward normal incidence) the angle between the fibre and the acoustic beam. Hence, this basic device geometry is adaptable to a wide range of frequencies, simply by choosing the IDT finger spacing and the fibre angle to correspond to the desired centre

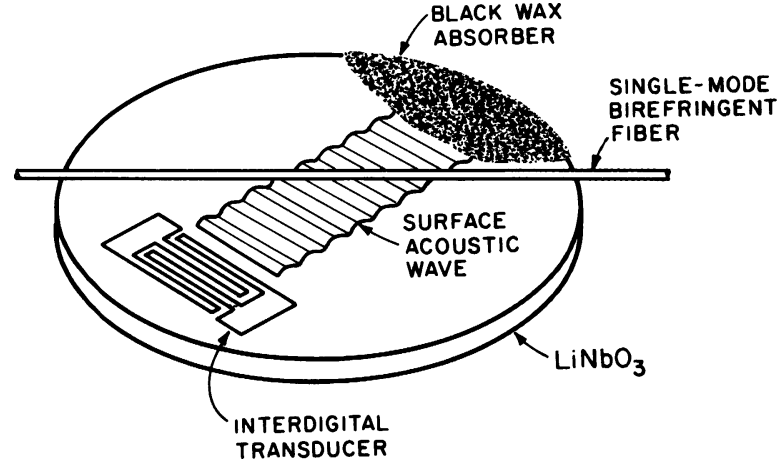

Fig. 10. - Next generation of frequency shifters, using surface waves on $\mathrm{LiNbO}_{3}$.

frequency. Although the angular alignment and straightness of the fibre become more critical at higher frequencies, we believe the requirements to be within reasonable limits.

\section{Conclusion.}

We have presented a novel technique for producing a single-sideband frequency shift in a light wave propagating in an optical fibre. This technique uses an acoustic wave to produce coupling between the two orthogonal polarizations of birefringent fibre. Light launched in one polarization can be coupled to the other polarization by an acoustic wave whose effective wavelength (as measured in the direction of the fibre) equals the fibre beat length. The light coupled from one polarization to the other is shifted in frequency by the frequency of the acoustic wave. Whether an upshift or a downshift is produced depends on whether the acoustic wave travels in the same or opposite direction as the light in the fibre and on which polarization is launched. Simple coupled-mode theory can be used to describe these features of the devices as well as the conditions for optimum coupling and the tuning bandwidth.

In our preliminary experiments, both surface acoustic waves and bulk longitudinal waves were used. With the surface wave device, in which the acoustic wave propagates in the same direction as the fibre, we were able to obtain a frequency shift of $1.5 \mathrm{MHz}$ and suppression of the unwanted sideband of $30 \mathrm{~dB}$. With the bulk wave device, in which the acoustic wave propagates at an angle to the fibre, we obtained a frequency shift of $15 \mathrm{MHz}$ and suppression of both the carrier and the unwanted sideband of greater than $20 \mathrm{~dB}$.

Future devices now under development will use surface waves launched on $\mathrm{LiNbO}_{3}$ interacting with the fibre at an angle to produce still higher frequency shifts, greater tunability, and higher conversion efficiency. We hope that these latter devices will perform well enough to justify their inclusion in a wide variety of sensor and interferometric systems. 


\section{References}

[1] Bergh, R. A., Digonnet, M. J. F., Lefevre, H. C., Newton, S. A. and Shaw, H. J., Single Mode Fiber Optic Components, in Fiber Optic Rotation Sensors, Editors : S. Ezekiel and H. J. Arditty (Springer-Verlag, New York) 1982.

[2] Stolen, R. H., Single-Mode Fiber Devices, Tech. Digest IEEE/OSA Seventh Topical Meeting on Integrated and Guided-Wave Optics, 1984.

[3] Nosu, K., Rashleigh, S. C., Taylor, H. F. and WeLlER, J. F., Acousto-optic Frequency Shifter for Single Mode Fibres, Electron. Lett. 19 (1983) 816.

[4] Brooks, J. L., Youngeuist, R. C., Kino, G. S. and Shaw, H. J., Active Polarization Coupler for Birefringent Fiber, Opt. Lett. 9 (1984) 249.

[5] Gloge, D., Weakly Guiding Fibers, Appl. Opt. 10 (1971) 2252.

[6] DyotT, R. B., Elliptically Cored Polarization Holding Fiber, in Fiber Optic Rotation Sensors, Editors : S. Ezekial and H. J. Arditty (Springer-Verlag, New York) 1982.

[7] Stolen, R. H., Ramaswamy, V., Kaiser, P. and Pleibel, W., Linear Polarization in Birefringent Single-mode Fibers, Appl. Phys. Lett. 33 (1978) 699.

[8] Ulrich, R. and Johnson, M., Single-Mode-FiberOptical Polarization Rotator, Appl. Opt. 18 (1979) 1857.
[9] Youngquist, R. C., Brooks, J. L. and Shaw, H. J., Birefringent-Fiber Polarization Coupler, Opt. Lett. 8 (1983) 656

[10] Rashleigh, S. C., Origins and Control of Polarization Effects in Single-Mode Fibers, IEEE J. Lightwave Tech. LT-1 (1983) 312.

[11] YarIv, A., Coupled-Mode Theory for Guided-Wave Optics, IEEE J. Quantum Electron. 9 (1973) 919.

[12] Stolen, R. H., Ashkin, A., Pleibel, W. and Dziedzic, J. M., In-line Fiber-Polarization-Rocking Rotator and Filter, Opt. Lett. 9 (1984) 300.

[13] Risk, W. P., Youngquist, R. C., Kino, G. S. and SHAW, H. J., Single-sideband Frequency Shifting in Birefringent Optical Fiber, to be published in the Proceedings of the 1984 SPIE Technical Symposium East (Arlington, VA), 478 (Fiber Optic and Laser Sensors).

[14] Varnham, M. P., Payne, D. N. and Love, J. D., Fundamental Limits to the Transmission of Linearly Polarized Light by Birefringent Fibres, Electron. Lett. 20 (1984) 55.

[15] Lardat, C. and Defranould, P., Applications of Edge-Bonded Transducers to SAW Components, Proc. IEEE 64 (1976) 627.

[16] Landau, L. D. and Lifshitz, E. M., Theory of Elasticity (Reading, Mass. : Addison-Wesley) 1959.

[17] KIM, B. Y., private communication. 